\title{
Journal of Oral and Maxillofacial Surgery
}

Copy of e-mail Notification

RUSH: Journal of Oral and Maxillofacial Surgery article <\# 52799> for proofing by Hita Iglesias

\section{$====$}

Dear Author,

The proof of your article, to be published by Elsevier in Journal of Oral and Maxillofacial Surgery, is available as a "PDF" file at the following URL:

http://rapidproof.cadmus.com/RapidProof/retrieval/index.jsp

Login: your e-mail address

Password: ----

The site contains 1 file. You will need to have Adobe Acrobat Reader software to read these files. This is free software and is available for user download at: http://www.adobe.com/products/acrobat/readstep.html

After accessing the PDF file, please:

1) Carefully proofread the entire article, including any tables, equations, figure legends and references.

2) Ensure that your affiliations and address are correct and complete.

3) Check that any Greek letter, especially "mu", has translated correctly;

4) Verify all scientific notations, drug dosages, and names and locations of manufacturers;

5) Be sure permission has been procured for any reprinted material.

6) Answer all author queries completely. They are listed on the last page of the proof;

You may choose to list the corrections (including the replies to any queries) in an e-mail and return to me using the "reply" button. Using this option, please refer to the line numbers on the proof. If, for any reason, this is not possible, mark the corrections and any other comments (including replies to questions) on a printout of the PDF file and fax this to Laura Dinkins-White(215-239-3388) or mail to the address given below.

Do not attempt to edit the PDF file (including adding < post-it $>$ type notes).

Within 48 hours, please return the following to the address given below:

1) Corrected PDF set of page proofs

2) Print quality hard copy figures for corrections if necessary (we CANNOT accept figures on disk at this stage). If your article contains color illustrations and you would like to receive proofs of these illustrations, please contact us within 48 hours.

If you have any problems or questions, please contact me. PLEASE ALWAYS INCLUDE YOUR ARTICLE NUMBER (located in the subject line of this e-mail) WITH ALL CORRESPONDENCE.

Sincerely,

Laura Dinkins-White

Senior Journal Manager

Elsevier Science

1600 John F.Kennedy Blvd.

Philadelphia, PA 19103-2899

Phone: 215-239-3375

Fax: 215-239-3388

la.white@elsevier.com 


\title{
Effectiveness of Chlorhexidine Gel Versus Chlorhexidine Rinse in Reducing Alveolar Osteitis in Mandibular Third Molar Surgery
}

\author{
Pilar Hita Iglesias, DDS, PhD, * Daniel Torres Lagares, DDS, PhD, $†$ \\ Rafael Flores Ruiz, DDS, $¥$ Natale Magallanes Abad, DDS, $\$ \\ Marta Basallote Gonzalez, DDS, | and \\ Jose Luis Gutierrez Perez, MD, DDSף
}

\begin{abstract}
Purpose: Chlorhexidine is an antimicrobial agent used in the prevention of postextraction alveolar osteitis, tooth decay and periodontal diseases.

There are various forms of chlorhexidine application. The most extensively studied is one that uses the rinse as the form of application.

Recently, a bioadhesive gel form has become available. Its main advantage is that it prolongs the bioavailability of chlorhexidine in the application area.

The purpose of this study was to compare the effectiveness of chlorhexidine gel versus a chlorhexidine rinse in reducing postoperative alveolar osteitis after the extraction of mandibular third molars.
\end{abstract}

Materials and Methods: The experimental or gel group $(n=41)$ applied the bioadhesive $0.2 \%$ chlorhexidine gel to the wound during the first postoperative week and a control or rinse group $(\mathrm{n}=$ 32 ) used a $0.12 \%$ chlorhexidine rinse during the first week postextraction.

Results: We observed a $70 \%$ decrease in postoperative alveolar osteitis in the gel group $(P=.040)$. The rinse group had $25 \%$ incidence postoperative alveolar osteitis, while the gel group had $7.5 \%$.

Conclusions: It was concluded that the topical application of bioadhesive chlorhexidine gel to the surgical wound during the postoperative week may decrease the incidence of alveolar osteitis after extraction of the mandibular third molars.

(C) 2007 American Association of Oral and Maxillofacial Surgeons

J Oral Maxillofac Surg xx:xxx, 2007

\begin{abstract}
Alveolar osteitis (AO) is a postextraction complication that was first defined by Crawford ${ }^{1}$ in 1896 . Throughout the years, various synonyms for AO have been used, such as alveolitis sicca dolorosa (dry socket), alveolalgia, osteomyelitis or fibrinolytic osteitis, pos-
\end{abstract}

\footnotetext{
${ }^{*}$ Department of Oral and Maxillofacial Surgery, University of Michigan, Ann Arbor, MI.

†Assistant Professor, Oral Surgery.

$\ddagger$ Master of Oral Surgery.

$§$ Master of Oral Surgery.

|Master of Oral Surgery.

ฯProfessor and Chairman.

Address correspondence and reprint requests to Dr Iglesias: University of Michigan, Oral and Maxillofacial Surgery, 1500 Medical Center Dr., Ann Arbor, MI 48103; e-mail: pilarh@umich.edu (c) 2007 American Association of Oral and Maxillofacial Surgeons $0278-2391 / 07 / x \times 0 x-0 \$ 32.00 / 0$ doi:10.1016/i.joms.2007.06.641
}

textraction osteomyelitis syndrome, fibrinolytic alveolitis, and localized alveolar osteitis. ${ }^{2-5}$

The most recent of these terms was provided by Blum, who defines $\mathrm{AO}$ as a postoperative pain in and around the dental alveolus, which increases in severity at some stage between the first and third day postextraction, accompanied by a partial or total disintegration of the intra-alveolar blood clot, and which may be accompanied by halitosis. ${ }^{6}$

The frequency of $\mathrm{AO}$ appearance ranges from $1 \%$ to $70 \%{ }^{6,7-12}$ The average rate of $\mathrm{AO}$ for all dental extractions is $3 \%$ to $4 \%$, according to various authors. ${ }^{7,13,14}$ The highest incidence generally occurs following the extraction of impacted third molars. In these cases, it may occur in $20 \%$ to $30 \%$ of these extractions, ${ }^{15-19}$ ie, 10 times more than for other dental extractions. ${ }^{6}$

Epidemiological studies linked to AO have identified various risk factors: difficulty of extraction, surgeon's inexperience, use of oral contraceptives, 
advanced age, female gender, smoking, immunosuppression, and surgical trauma. ${ }^{18,20}$

AO causes increased psychological harm to the patient and the health professionals, because the painful symptoms which accompany this pathology are extremely uncomfortable for the patient. $^{21}$

Chlorhexidine (CHX) is a biguanide antiseptic agent that has been proven effective in the prevention of $\mathrm{AO}$ in the form of a mouth rinse and bioadhesive gel. The method of administration of this gel has the main advantage of providing a greater bioavailability in the application area, and therefore the medication has a more prolonged release. ${ }^{12,21}$ The objective of this study is to compare the effectiveness of these two forms of $\mathrm{CHX}$ ( $0.2 \%$ bioadhesive gel and $0.12 \%$ rinse) in the prevention of postoperative AO after the extraction of retained third molars, by means of topical application to the wound during the 7 days after the intervention.

\section{Materials and Methods}

This clinical study was a randomized, prospective clinical trial with parallel groups in a single center. It was carried out in the Faculty of Odontology at the University of Seville and in the Oral and Maxillofacial Surgery Service of the Virgen de Rocio University Hospitals, Seville. The study involved the treatment of 73 patients of both genders, between the ages of 18 and 60 years old, from June 2005 to November 2005. These patients presented with a mandibular third molar with a difficulty index ranging between 4 and 7 , inclusive, according to the Koerner scale. ${ }^{22}$ The degree of difficulty was rated by a single investigator who performed all preoperative patient selection. Extraction of the molar was required. The patients could not take any type of antibiotic or analgesic in the 4 days preceding the procedure. Exclusion criteria included the following: nonfulfillment of 1 or all of the inclusion criteria, patients with any other disease which would contraindicate oral surgery, patients with AIDS or any type of immunosuppression, pregnant or lactating women, patients allergic to chlorhexidine, articaine, paracetamol or ibuprofen, patients in whom the administration of epinephrine is contraindicated, patients who required the simultaneous extraction of two wisdom teeth, patients who presented with a jawbone associated pathology, patients in whom the extraction of the retained wisdom tooth lasted for more than 30 minutes and noncooperative patients (psychic-motor dysfunction and behavior disorders).

All of the patients in the study gave their informed consent, and were covered by public liability insurance. The study was approved by the Ethics Committee of the University of Seville and followed the principles of the Helsinki Declaration.
Two pharmaceutical forms of CHX were studied: $0.2 \%$ bioadhesive gel and $0.12 \%$ mouth rinse (Laboratorios Lácer SA, Barcelona, Spain). The aim was to compare the decrease in postoperative AO incidence among the group of patients who received application of CHX bioadhesive gel, and those who received CHX rinse, both in the topical form, during the postoperative period after surgery to extract the mandibular third molar.

The patients underwent intervention of the inferior alveolar nerve and buccal nerve under local anesthesia (articaine 4\% epinephrine, Laboratorios Inibsa, Barcelona, Spain). A bayonet incision was performed in order to gain access to the wisdom tooth, carrying out osteotomy in all cases and dental sectioning when necessary. Once the tooth had been extracted, the alveolus was cleaned, the bone edges were smoothed, and bioadhesive $0.2 \%$ chlorhexidine gel was applied inside the alveolus. Finally, the wound was sutured with simple $4 / 0$ silk stitches.

The patients were randomly classified into 2 groups by means of a simple allocation using a computer program: the gel group and the rinse group, according to the pharmaceutical form of CHX used during the postoperative period. Having carried out the procedure, the envelope corresponding to the patient code was opened, and this indicated the group to which the patient had been assigned. By way of postoperative treatment, the patients took $600 \mathrm{mg}$ ibuprofen (1 tablet every 8 hours) and $500 \mathrm{mg}$ of $14.05 \mathrm{mg}$ paracetamol codeine (1 tablet every 8 hours; Cod-efferalgan ${ }^{\circledR}$, Bristol-Myers-Squibb, Madrid, Spain).

The patients in the gel group continued topical treatment with bioadhesive $\mathrm{CHX}$ gel, applied to the surgical wound twice a day (morning and night time) during the first postoperative week, beginning on the same day as the intervention. The patients in the rinse group continued topical treatment with $\mathrm{CHX}$ mouthwash, used twice a day (morning and night time) during the postoperative week, beginning on the same day as the intervention.

The independent variable was the application of CHX bioadhesive gel or CHX rinse during the postoperative period. The main dependent variable was the appearance of postoperative AO according to Blum's standardized criteria.

Subjects were evaluated on the third and seventh postoperative day. Diagnosis of AO was considered positive when patients presented with postoperative pain in and around the dental alveolus, that had increased in severity sometime between the first and third postoperative day, and was accompanied by partial or total loss of the intra-alveolar blood clot. In addition, the link between the appearance of postoperative $\mathrm{AO}$ and the risk variables described in the 
literature were analyzed: age, gender, smoking, oral contraceptives, and degree of difficulty of extraction.

All clinical assessment was carried by a single blind investigator, trained by the directors of this study in previous studies sharing the same $\mathrm{AO}$ criteria. All study subjects reported using the medication prescribed to them. Compliance assessment was achieved by intention-to-treat analysis. Tolerance to the treatment, defined as the frequency that patients developed 1 or more adverse effects was assessed on a verbal score of 1 (maximum tolerance) to 5 (minimum tolerance or maximum intolerance) during the third and seventh postoperative day. The $\chi^{2}$ test was applied for the comparison of the proportions between the 2 groups (gel and rinse), and Student $t$ test for the comparison of the mean values in quantitative variables.

\section{Results}

A total of 73 patients underwent intervention (73 mandibular third molars). There were 41 patients in the gel group and 32 patients in the rinse group. The progress of a total of 70 were followed until the end of the study, because 2 patients from the gel group and 1 from the rinse group did not complete the study. The average age was 29 years old (ranging from 18 to 59). Fifty-four patients were female and 19 were male. Eight women were taking oral contraceptives. Twenty-nine patients were smokers (16 females and 10 males). Details of both groups regarding age, gender, smoking habits, use of oral contraceptives, and degree of difficulty are displayed in Table 1 (significant statistical differences were not found between the two groups).

In the gel group, $7.5 \%$ of $\mathrm{AO}$ incidence was found, while in the mouthwash group, there was $25 \%$; the difference was statistically significant, $P=.040$ for the $\chi^{2}$ test (Table 1 ). None of the patients displayed adverse effects to the treatment, and there was adequate tolerance in both groups.

\section{Discussion}

Currently, there are two main etiopathogenic theories about AO: Birn's fibrinolytic theory and the bacterial theory. ${ }^{3,15-19,21}$ With respect to these etiopathogenic theories, the prevalence of one theory over the other has not been generally accepted, as there is no conclusive data to definitively reject or accept one of the two. The origin of AO probably lies in an interactive mix of both theories.

Epidemiological studies related to $\mathrm{AO}$ have identified various risk factors in the development of $\mathrm{AO}$, mainly: difficulty of extraction, surgeon's inexperience, use of oral contraceptives, advanced age, fe-

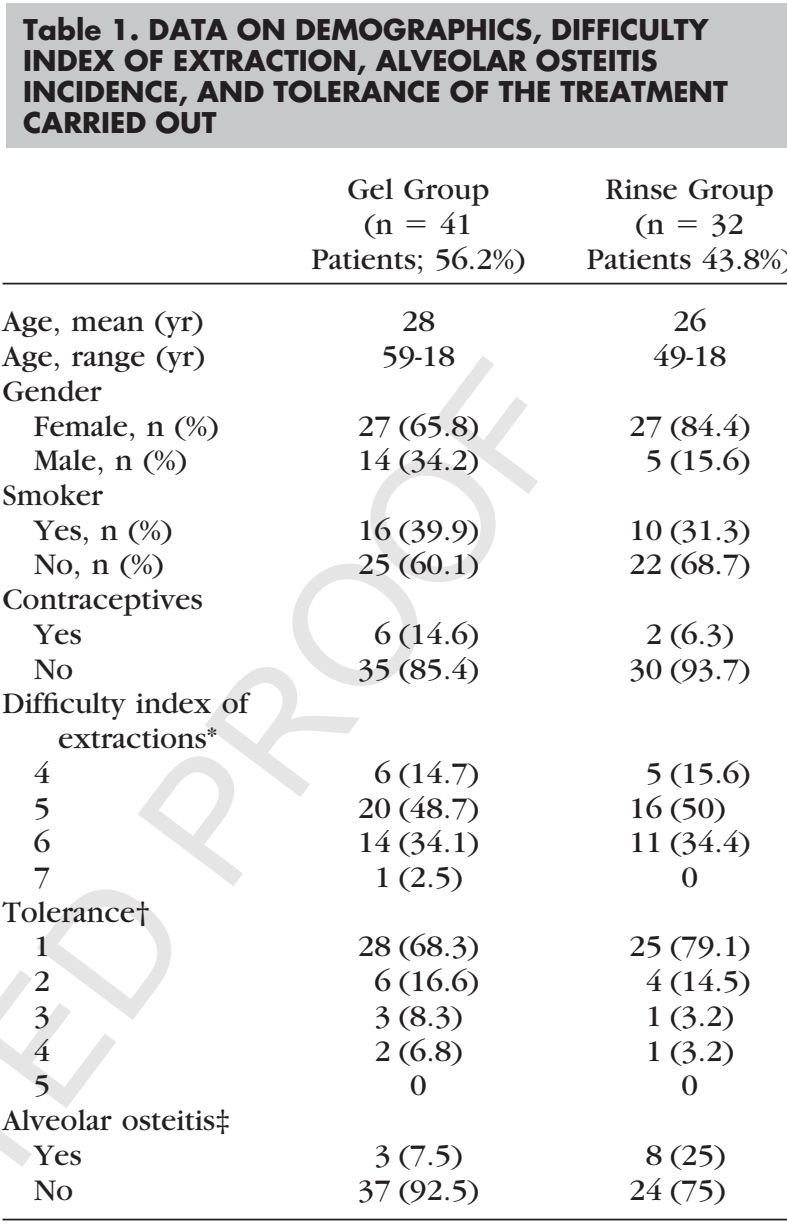

Data are $\mathrm{n}(\%)$ except where noted.

*๑॰

†Verbal scale from 1 meaning totally tolerable, to 5 meaning totally intolerable.

$\ddagger$ Student $t$ test, $P=.040$.

Iglesias et al. Chlorhexidine Gel vs Rinse and Osteitis. J Oral Maxillofac Surg 2007.

male gender, smoking, immunosuppression, and surgical trauma. ${ }^{18,22}$

The preventative measures taken in the therapeutic management of $\mathrm{AO}$ are summarized as follows: washing with saline solution, eugenol dressings to provide relief, antifibrinolytic agents, antibiotics, and antiseptic agents. These last 2 measures are, probably, those that have had the most success in the prevention of AO. ${ }^{20,23,24,29}$ Antibiotics are more expensive, may create resistance and their efficiency in the prevention of $\mathrm{AO}$ has been questioned by several authors. ${ }^{19}$

The contributions which support the validity of CHX in the control of bacterial plaque ${ }^{25}$ and the relation between oral hygiene and the prevention of alveolitis sicca ${ }^{26}$ have been numerous to date.

CHX has been shown to be a good preventative agent of AO. Various application protocols have been studied, both in mouth rinse and in the postoperative intra-alveolar application of bioadhesive gel, although 
there are no studies which simultaneously compare the effectiveness of $\mathrm{CHX}$ bioadhesive gel with $\mathrm{CHX}$ rinse following the extraction of retained mandibular third molars.

In a recent meta-analysis by Caso et $\mathrm{al}^{21}$ on the use of CHX on impacted third molars in the postoperative period, the authors concluded that the use of $\mathrm{CHX}$ mouth rinse from the day of intervention and during the postoperative period produce a decrease in $\mathrm{AO}$ incidence. The minimum postoperative period of time during which mouthwash should be applied could not be determined in this study.

Berwick and Lessin ${ }^{17}$ did not discover differences in $\mathrm{AO}$ incidence in the 2 study groups ( $\mathrm{CHX} 0.12 \%$ and cetylpyridinium $0.05 \%$ ). Delilbasi et $\mathrm{al}^{27}$ found similar percentages of $\mathrm{AO}$, using $0.2 \% \mathrm{CHX}$ mouth rinse and saline solution. Ragno and Szkutnik ${ }^{16}$ discovered a $17.5 \%$ decrease in the group that used $0.2 \%$ CHX mouth rinse after the extraction of retained third molars compared with $36 \%$ of $\mathrm{AO}$ in the control group (placebo).

Larsen $^{28}$ found $16 \%$ of $\mathrm{AO}$ in the control group (placebo) compared with $8 \%$ in the experimental group $(0.12 \% \mathrm{CHX}$ mouth rinse during the pre and postoperative weeks). Other authors have found a $50 \%$ decrease of AO incidence using $0.12 \% \mathrm{CHX}$ mouth rinse. Torres et $\mathrm{al}^{29}$ discovered an $11 \%$ decrease of $\mathrm{AO}$ in the experimental group (intra-alveolar bioadhesive $0.2 \% \mathrm{CHX}$ gel) after the extraction of retained mandibular third molars, compared with 30\% in the experimental group (intra-alveolar medication versus placebo).

The only study that we have found which compares various proportions of chlorhexidine did not show significant differences between the $0.1 \%$ and $0.2 \%$ CHX groups. There was a significant improvement between both chlorhexidine groups and the control group. ${ }^{25}$ Although the concentration of chlorhexidine was different in the gel group and in the mouthwash group, previous data supports the comparability of both groups.

The number of patients in this study is sufficient to assess the main variable: appearance or absence of $\mathrm{AO}$ postextraction. In other studies, this number ranges between 20 and 67 per group. ${ }^{15,17,18,25,27}$ The average age of the patients was higher than that obtained by other authors. With respect to the proportion of males/females, there are studies such as those by Torres et $\mathrm{al}^{29}$ and Hermesch et al, ${ }^{30}$ which coincide with our data, while other authors had a similar proportion of males/females ${ }^{30}$ in their studies. With respect to the use of oral contraceptives (14\%), we obtained similar results to those of Torres et al. ${ }^{29}$ In other studies such as those by Larsen ${ }^{28}$ and Hermesch et $a 1,{ }^{30}$ the results showed percentages of $53 \%$ and $32 \%$ respectively for women who were taking the oral contraceptives, while in the study by Bonine, ${ }^{31}$ this value did not surpass $6 \%$.

The percentage of patients who smoked, was higher than in studies by other authors such as Torres et $\mathrm{al}^{29}$ (25\% of smokers in their sample) and Larsen ${ }^{28}$ (28.16\% of smokers). Nevertheless, these percentages are within the limits found in other literature (16.3\%, $15.12 \%$ and $25.42 \%){ }^{27,30,31}$ We have not found significant statistical differences in $\mathrm{AO}$ incidence in groups of smokers and nonsmokers, nor have we found significant differences between patients who take contraceptives and those who do not.

In the experimental group (gel), we found a statistically significant decrease $(70 \%, P=.05)$ in the incidence of postoperative $\mathrm{AO}$ compared with the mouthwash group. These results may be explained by the bioadhesive properties of the gel, which prolong the release of $\mathrm{CHX}$ at the application site. No adverse effect was recorded in the patients treated, as referred to in other similar studies. ${ }^{31}$

The results of this clinical study show that the application of bioadhesive $0.2 \% \mathrm{CHX}$ gel to the postoperative wound after the extraction of retained mandibular third molars decreases AO incidence compared with the application of $0.12 \% \mathrm{CHX}$ mouthwash under similar circumstances.

\section{Acknowledgments}

The authors thank Laboratorios Lacer Barcelona España, for the donation of chlorhexidine samples used in this study.

\section{References}

1. Crawford JY: Dry socket. Dent Cosmos 38:929, 1986

2. Swanson AE: Prevention of dry socket: An overview. Oral Surg Oral Med Oral Pathol 70:131, 1990

3. Swanson AE: A double-blind study on effectiveness of tetracycline in reducing the incidence of fibrinolytic alveolitis. J Oral Maxillofac Surg 47:165, 1989

4. Moore JW, Brekke JH: Foreign body giant cell reaction related to placement of tetracycline-treated polylactic acid: Reported of 18 cases. J Oral Maxillofac Surg 48:808, 1990

5. Trieger N, Schlagel GD: Preventing dry socket. A simple procedure that works. J Am Dent Assoc 2:67, 1991

6. Blum IR: Contemporary views on dry socket (alveolar osteitis): A clinical appraisal of standardization, aetiopathogenesis and management: A critical review. Int J Oral Maxillofac Surg 31: 309, 2002

7. García Murcia MJ, Peñarrocha Diago M: Alveolitis seca: Revisión de la literatura y metaanálisis. Rev Actual Odontoestomatol Esp 44:25, 1994

8. Barclay JK: Metronidazole and dry socket: Prophylactic use in mandibular third molar removal complicated by non-acute pericoronitis. N Z Dent J 83:71, 1987

9. Fridrich KL, Olson RA: Alveolar osteitis following surgical removal of mandibular third molars. Anesth Prog 37:32, 1990

10. Rozanis J, Schofield ID, Warren BA: Is dry socket preventable? Dent J 43:233, 1977

11. Schow SR: Evaluation of postoperative localized osteitis in mandibular third molar surgery. Oral Surg Oral Med Oral Pathol 38:352, 1974

12. Ariza E, González J, Boneu F, Hueto JA, Raspall G: Incidencia de la alveolitis seca, tras la exodoncia quirúrgica de terceros mo- 
lares mandibulares en nuestra Unidad de Cirugía Oral. Rev Esp Cir Oral Maxilofac 21:214, 1999

13. Rood JP, Murgatroyd J: Metronidazole in the prevention of "dry socket." Br J Oral Surg 17:62, 1979

14. Jaafar N, Nor GM: The prevalence of post-extraction complications in an outpatient dental clinic in Kuala Lumpur Malaysia: A retrospective survey. Singapore Dent J 23:24, 2000

15. Larsen PE: The effect of a chlorhexidine rinse on the incidence of alveolar osteitis following the surgical removal of impacted mandibular third molar. J Oral Maxillofac Surg 49:932, 1991

16. Ragno JR Jr, Szkutnik AJ: Evaluation of $0.12 \%$ chlorhexidine rinse on the prevention of alveolar osteitits. Oral Surg Oral Med Oral Pathol 72:524, 1991

17. Berwick JE, Lessin ME: Effects of a chlorhexidine gluconate oral rinse on the incidence of alveolar osteitis in mandibular third molar surgery. J Oral Maxillofac Surg 48:444, 1990

18. Larsen PE: Alveolar osteitis after surgical removal of impacted mandibular third molars. Identification of the patient at risk. Oral Surg Oral Med Oral Pathol 73:393, 1992

19. Ritzau M, Hillerup S, Branebjerg PE, Ersbol BK: Does metronidazole prevent alveolitis sicca dolorosa? A double-blind, placebo-controlled clinical study. J Oral Maxillofac Surg 21:299, 1992

20. Bloomer CR: Alveolar osteitis prevention by immediate placement of medicated packing. Oral Surg Oral Med Oral Pathol Oral Radiol Endod 90:282, 2000

21. Caso A, Hung LK, Beirne OR: Prevention of alveolar osteitis with clorhexidine: A meta-analytic review. Oral Surg Oral Med Oral Pathol Oral Radiol Endod 99:155, 2005

22. Koerner KR: The removal of impacted third molars. Principles and procedures. Dent Clin North Am 38:255, 1994

23. Sweet JB, Butler DP: Increased incidence of postoperative localized osteitis in mandibular third molar surgery associated with patients using oral contraceptives. Am J Obstet Gynecol 127:518, 1977

24. Sweet JB, Butler DP: Predisposing and operative factors: Effect on the incidence of localized osteitis in mandibular third-molar surgery. Oral Surg Oral Med Oral Pathol 46:206, 1978

25. Fotos PG, Koorbusch GF, Sarasin DS, Kilt RJ: Evaluation of intra-alveolar chlorhexidine dressings alter removal of impacted mandibular third molars. Oral Surg Oral Med Oral Pathol 73:383, 1992

26. Tjernberg A: Influence of oral hygiene measures on the development of alveolitis sicca dolorosa after surgical removal of mandibular third molar. Int J Oral Surg 8:430, 1979

27. Delilbasi C, Saracoglu U, Keskin A: Effects of $0.2 \%$ chlorhexidine gluconate and amoxicillin plus clavulanic acid on the prevention of alveolar osteitis following mandibular third molar extractions. Oral Surg Oral Med Oral Pathol Oral Radiol Endod 94:301, 2002

28. Larsen PE: Use of chlorhexidine to prevent alveolar osteitis. J Oral Maxillofac Surg 48:1244, 1990

29. Torres D, Gutiérrez JL, Infante P, García-Calderón M, Romero MM, Serrera-Figallo MA: Randomized, double-blind study on effectiveness of intra-alveolar clorhexidine gel in reducing the incidence of alveolar osteitis in mandibular third molar surgery. Int J Oral Maxillofac Surg 35:348, 2006

30. Hermesch CB, Hilton TJ, Biesbrock AR, et al: Perioperative use of $0.12 \%$ chlorhexidine gluconate for the prevention of alveolar osteitis. Efficacy and risk factor analysis. Oral Surg Oral Med Oral Pathol Oral Radiol Endod 85:381, 1998

31. Bonine FL: Effect of chlorhexidine rinse on the incidence of dry socket in impacted mandibular third molar extraction sites. Oral Surg Oral Med Oral Pathol Oral Radiol Endod 79:154, 1995 


\section{AUTHOR QUERIES}

\section{AUTHOR PLEASE ANSWER ALL QUERIES}

AQ9 - Please confirm 68.7\% is correct for non-smoking rinse group.

AQ10 - For Tolerance, and Alveolar osteitis, please add comment in footnote as to why the number of patients in the gel group do not add to 41, and for Tolerance, rinse group, why the number of patients does not add to 32. Please check that percentages are correct for these categories.

AQ11 - Please add to * footnote: Explanation of the scale used for difficulty index of extractions. Please note "number of teeth" was removed from heading; if needed, please clarify meaning of the phrase (eg, number (quantity) of teeth extracted, number of the tooth that was extracted, order in which the teeth were removed, or otherwise).

AQ1 - Please approve or modify short title for running head (must be $<45$ characters, including spaces).

AQ2 - Please provide complete affiliation information for each author: position (eg, Professor), Department(s), institutional affiliation, and location (city, state, and country if not USA).

AQ3 - Structured abstract headings are required, per journal style, and have thus been added. Please approve or modify placement.

AQ4- Date of publication for Crawford in reference list is 1986; is 1896 meant here?

AQ5- Please confirm AO meant here.

AQ6 - 'Conclusion' is never allowed as a heading, per journal style, and has thus been deleted.

AQ7- Unable to verify journal on PubMed, or article with this title by this author; please confirm it is correct.

AQ8 - Unable to verify reference on PubMed, using search of journal and first author; please confirm it is correct. 